\title{
Optimization of Power and Area in Multipliers for Image Processing Applications
}

\author{
V. Keral Shalini, S. Keerthika Devi, P. Monisha and M. Madhumitha \\ Electronics and Communication Engineering, M. Kumarasamy College of Engineering, Karur - 639113, \\ Tamil Nadu, India; shalini.keral14@gmail.com, keerthika.2897@gmail.com, \\ monishaparamasivam6@gmail.com, krishnapriyamani1979@gmail.com
}

\begin{abstract}
Objectives: This paper is aimed to find a multiplier to provide a physically compact high speed and low power consumption unit. Being a core part of arithmetic processing unit multipliers are in extremely high demand on its speed and low power consumption. Multiplier plays an important role in today's digital image processing and various other applications. Methods/Statistical Analysis: In modern embedded electronics devices, power consumption is a first-class design concern. Hardware-level approximation mainly targets arithmetic units, such as adders and multipliers, widely used in portable devices to implement multimedia algorithms, e.g., image and video processing. Findings: Compressor has 5 inputs A, B, C, D and $C_{\text {in }}$ to create 3 outputs Sum, Carry and $C_{\text {out }}$. The 4 inputs A, B, C and D and the output Sum has identical weight. The input $\mathrm{C}_{\text {in }}$ is output from a preceding lower widespread compressor and the $\mathrm{C}_{\text {out }}$ output is for the compressor inside the next significant level. Application/Improvements: The approximation multiplier is used to improve the speed and efficiency. It is used to reduce the area and time consumption. It is widely used in the digital image processing, FIR filters, reduce the multiplication procedure.
\end{abstract}

Keywords: Approximation Multiplier, Adder, Model Sim, Shifter, Multiplexer

\section{Introduction}

Multipliers assume an imperative part in the present computerized flag preparing and different applications. With pushes in advancement, various scholars have endeavored and are demanding to enterprise multipliers which deals both of the going with layout goals - quick, low power consumption use, typicality of configuration and consequently fewer domain blend of them in one of the MUX thusly assembling sensible several fast, minimum voltage and littler VLSI utilization.

In this section, the related research in the field of hardware approximate computing is discussed. General purpose approximation techniques applied to any arithmetic circuit and circuit-specific approximation either to adder or multiplier designs. ${ }^{1-5}$. This multiplicand split into three $m$-bit segments and perform the multiplication utilizing the segment containing the MSB as 1which is used to perform the $n \times n$ multiplication.

\subsection{Serial Multiplier}

Nonetheless it is conceivable additionally, to utilize pressure procedure then the quantity of incomplete items can be lessened before option is performed.

The option of the PPs are executed as the moderate estimations of PPs option remain put away in the DFF, coursed besides included through the recently framed PP.

Figure 1 defines about the working of serial multiplier. It is used to increase the efficiency of multiplier by working efficiently. 


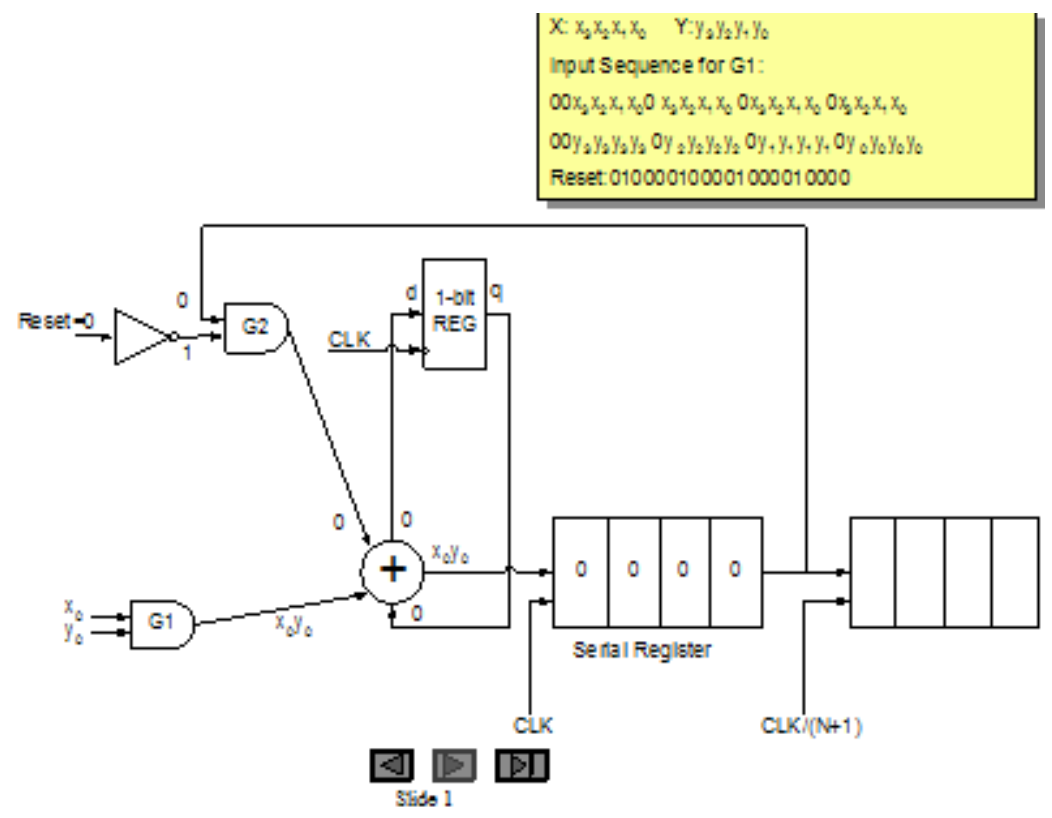

Figure 1. Serial multiplier.

\section{Block Diagram}

The Figure 2 shows the block diagram of the Approximation multiplier.

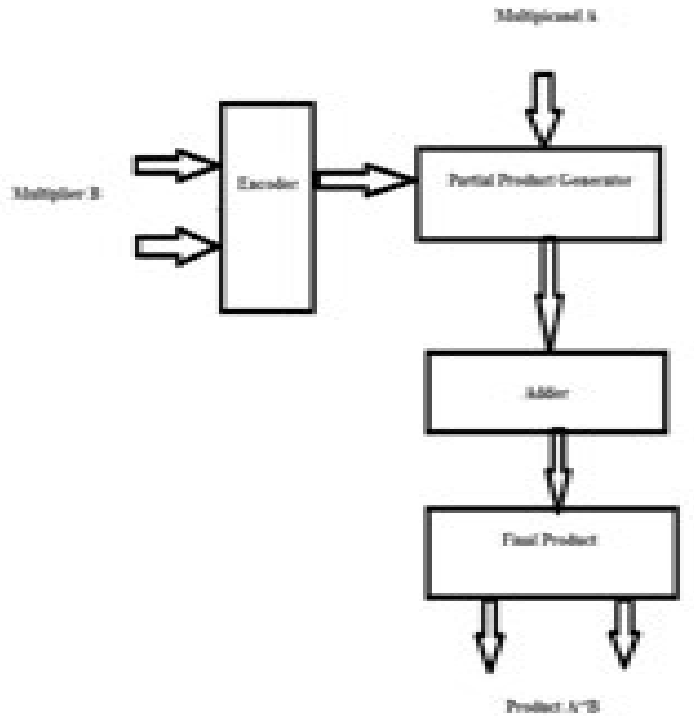

Figure 2. Approximation Multiplier.

\subsection{Serial and Parallel Multiplier}

The universal outline of the serial or parallel multiplier is showed up in the design underneath. One operand is supported to the circuit in parallel while the further is serial.

\subsection{Add AND Shift Multiplier}

Universal building that move besides incorporate multiplier was showed up at the design underneath on behalf of a 32 bit increment. The Figure 3 shows the estimation of MUX LSB bit, an estimation that multiplicand is incorporated an amassed. An every clock cycle of multiplier was moved one side of the other side besides it is regard attempted. If it is 0 , the action is accomplished. If it is 1 , the multiplicand will add. Moved through one side to other side.

Subsequently each and every multiplier bits has been attempted thing in the authority. This circuit has a couple of inclinations in odd circuits.

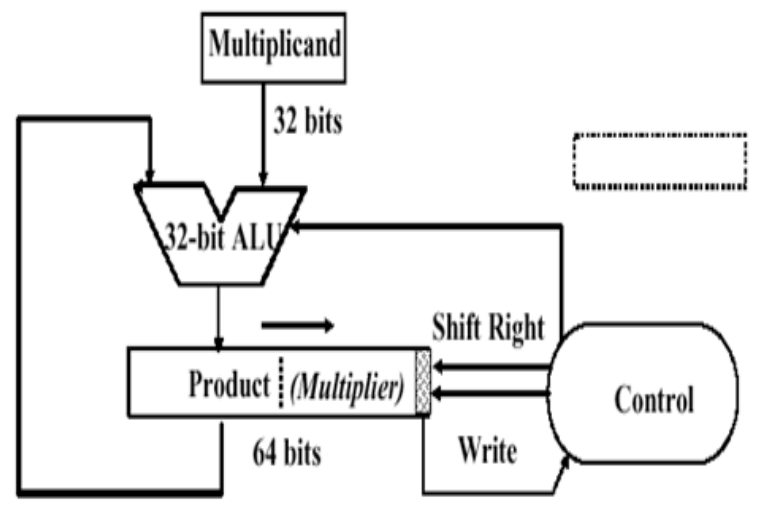

Figure 3. Shift and add Multiplier. 


\section{Array Multipliers}

Group multiplier is extraordinary in light of its ordinary structure. Multiplier circuit relies upon incorporate and move estimation. Each fragmented thing is finished by development of multiplicand with one MUX of the bit. Incomplete thing is relocated by the bit requests after that additional.

The Figure 4 shows the Augmentation algorithm. Augmentation could be accomplished add to ordinary authorization on incite snake. $\mathrm{N}-1$ adders were essential which $\mathrm{N}$ is the multiplier length which has been used.

In spite of the way that the strategy is clear as it can be realized through this case, the extension is completed serially and furthermore in parallel. In order to advance the interruption and territory the CRA's are supplanted add the Carry Save Adders, in that each convey, total flag are accepted towards the adders will following phase. Last item stays acquired in a last snake by somewhat quick viper (more often than not convey swell viper). The Figure 5 shows the working of Full Adder in complete manner. In exhibit duplication we have to include, the same number of halfway items as there are multiplier bits.

$$
\text { Total Area }=(\mathrm{N}-1)^{\star} \mathrm{M}^{\star} \text { Area }_{\text {FA }}
$$

$$
\text { Delay= } 2(\mathrm{M}-1) \square_{\mathrm{FA}}
$$

By and by mutually A and B might sure undesirable; Two's complement numeral method was implied address those.

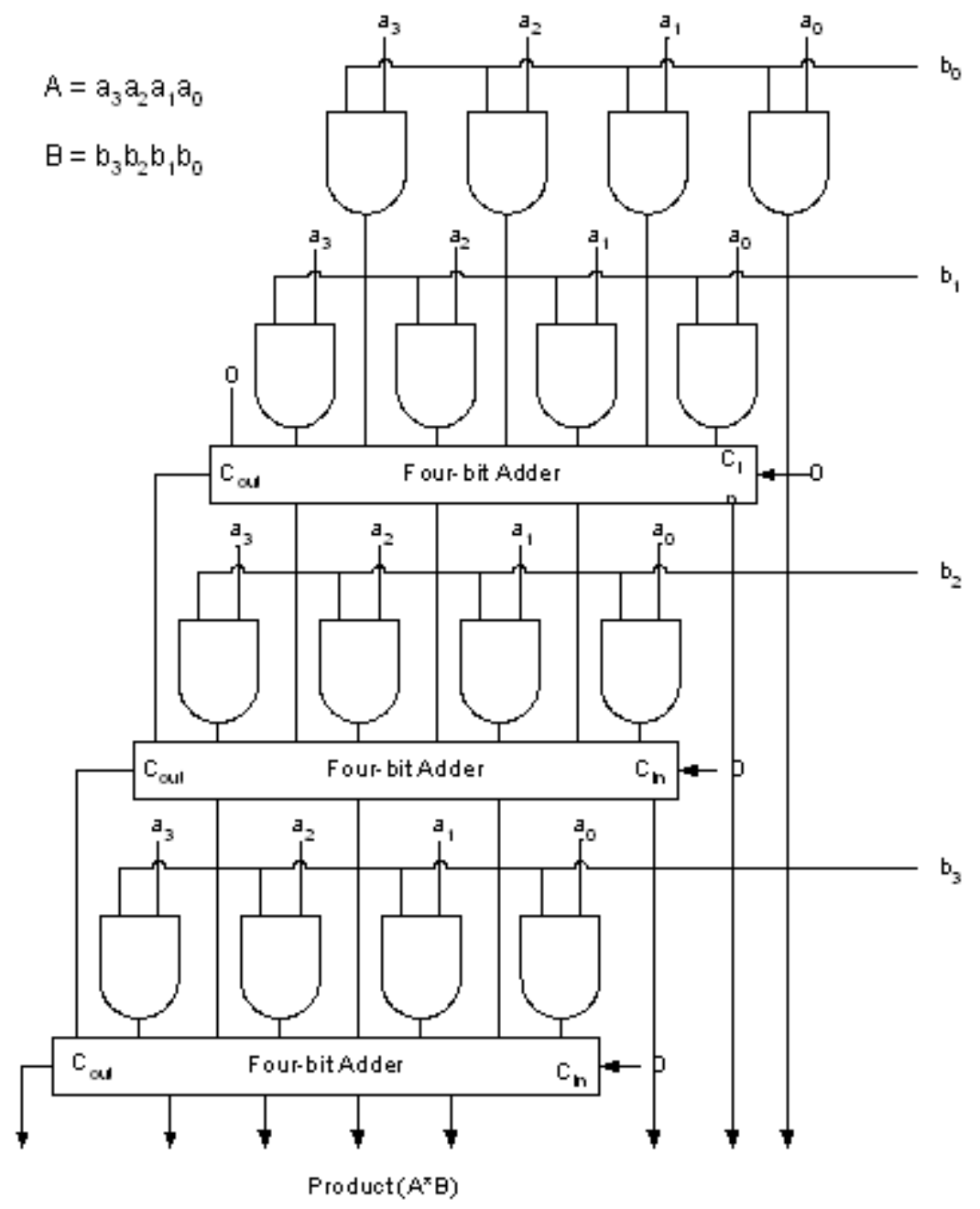

Figure 4. Array Multiplier. 


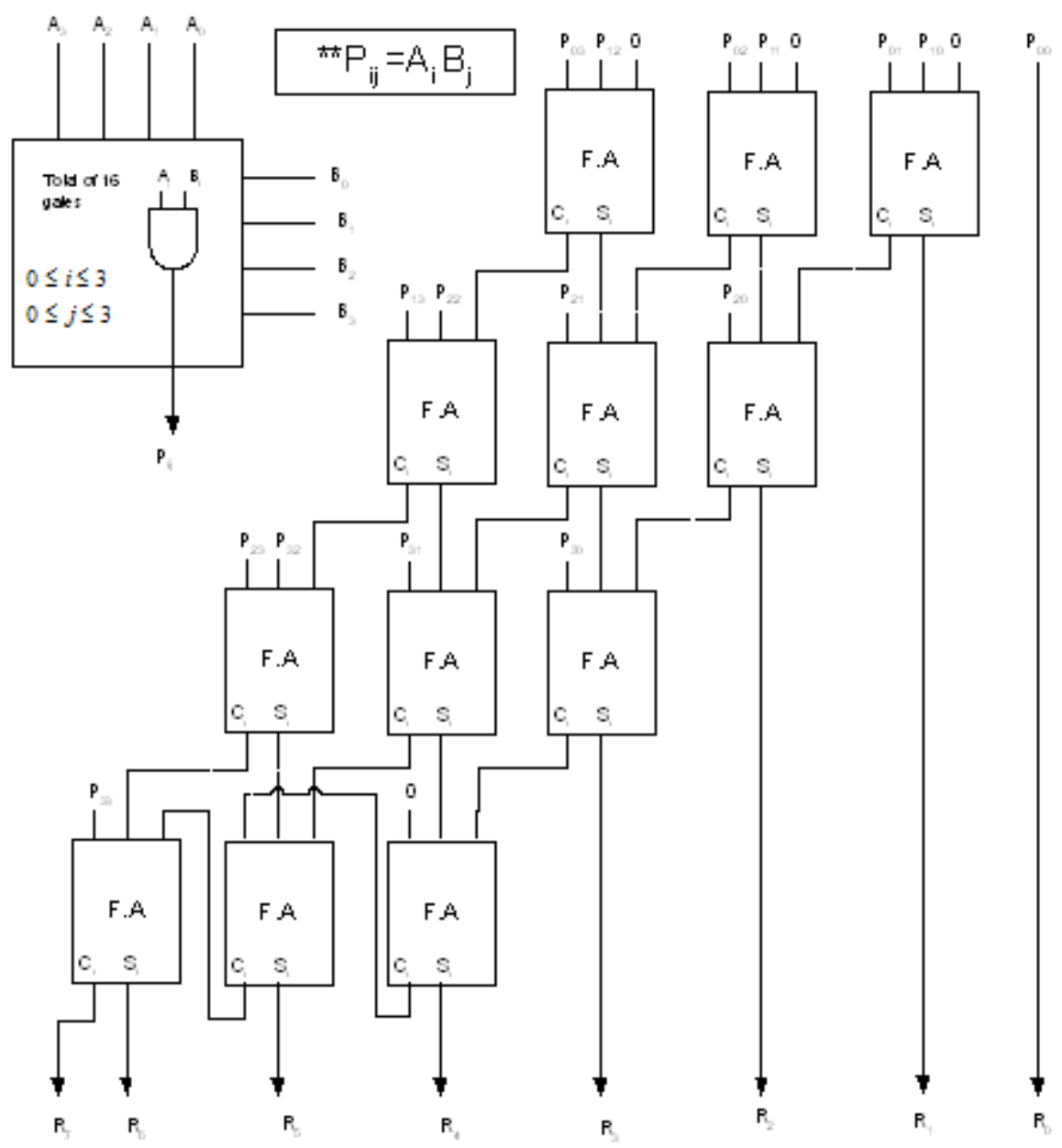

Figure 5. Full adder using Multipliers.

In case the multiplier operand exists certain then fundamentally a comparable strategy could be implemented yet mind must be occupied for mark piece development. The clarification behind overseeing checked number erroneously is the nonappearance of mark piece development in this multiplier.

\begin{tabular}{ll}
$a 1 a 0$ & $a 1 a 0$ \\
$X \quad b 1$ bo & $X$ b1 b0 \\
\hline$a 1 b 0$ a0b0 & alb0 a1b0a1b0 a0b0 \\
a1b1 a0b1 & alb1 a1b1 a0b1 \\
Wrong & Correct
\end{tabular}

The Figure 6 shows an approach manages adjust that blame, that don't have grow the greater part of the bits in the halfway thing augmentation.

At the point when 2's supplement incomplete items are included convey spare math all numbers to be included one viper arrange must be of equivalent piece length. Consequently, the sign bit of the fragmented product in the principle line besides total in addition to pass on indications of every snake push are prolonged upto utmost basic sign bit of the number with the greatest preeminent motivating force to be incorporated this stage. The sign piece development realizes and advanced fan out of the sign piece signal appeared differently in relation to the pile of various signs and as necessities be backs off the speed of the circuit.

Calculations live while including bi halfway items $(A+B)$ which will dispose of the definition piece expansion (Please observe Appendix A which two numbers will certain undesirable):

1. Expand value piece of $A$ and one piece then transform this broadened digit. 


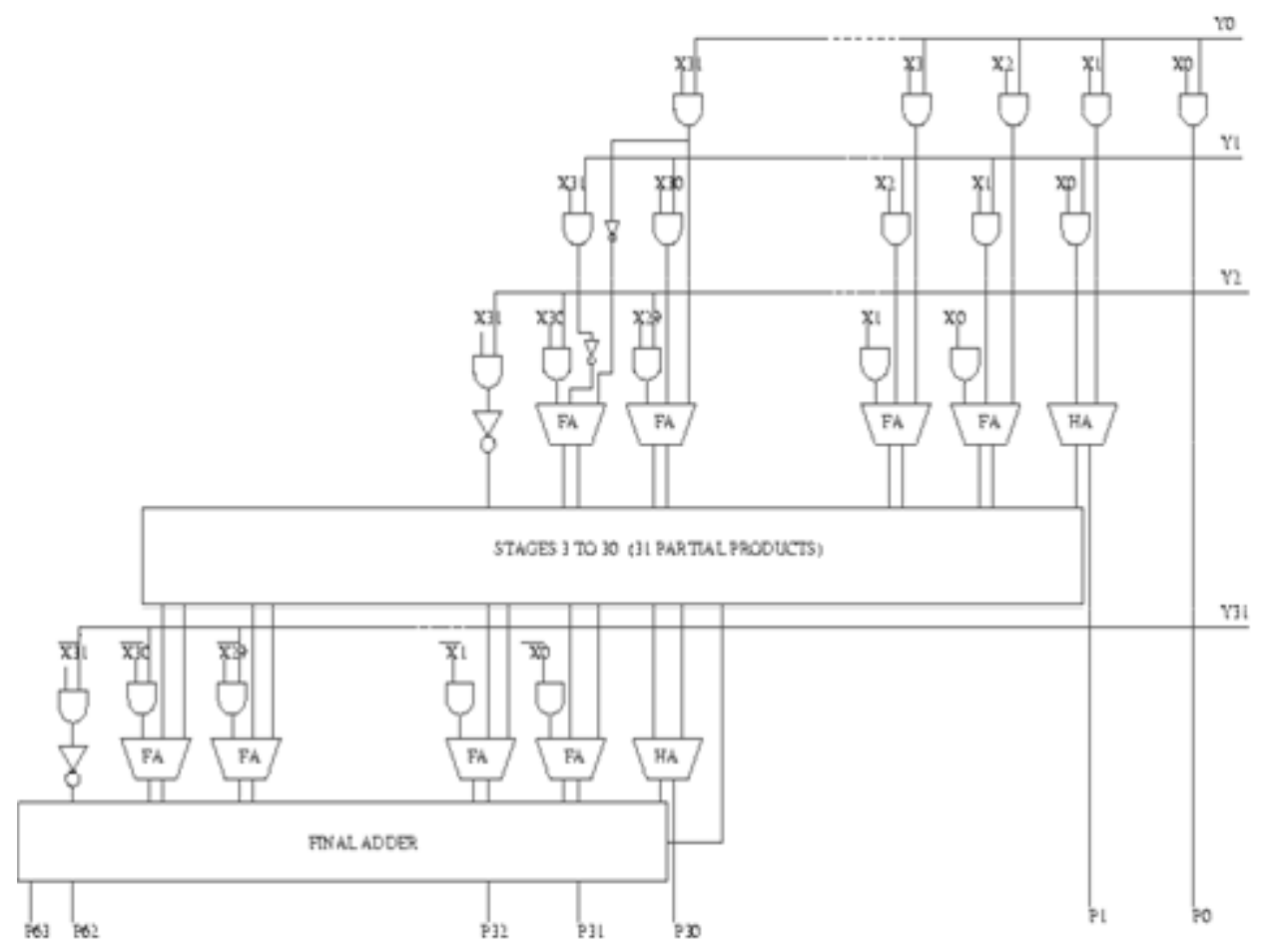

Figure 6. Array Multiplier for a 32 bit number(2"s complement number.

2. Transform sign piece of $B$.

3. Include $A$ in addition to $B$. Include one to one position left MSB of B.

\section{Booth Multipliers}

This is capable calculation on behalf of marking curtain augmentation, which considers both affirmative and undesirable numbers reliably. For the typical incorporate move action, every multiplier bit makes one a few of the multiplicand to be used to the inadequate thing. In event that the multipliers is huge, at that point countless must be included. For this circumstance the deferment of multiplier is settled fundamentally by the amount of increases to be performed. In the event that there is an approach to diminish the quantity of the augmentations, the execution will show signs of improvement.

Corner computation was a framework which will diminish measure of multiplicands things. The degree of number to be tended to, the highest delineation radices prompt less bit. As a k-bit two fold number can be deciphered as K/2-digit radcies-4 number, a K/3-digit radices- 8 number, and so forth, this can manage in excess of one piece multiplier in each utilizing high radices increase. This will appeared for Radices-4 to the circumstance underneath.

\begin{tabular}{lll} 
Multiplicand & $\mathrm{A}=$ & $\bullet \bullet \bullet$ \\
Multiplier & $\mathrm{B}=$ & $(\bullet \bullet)(\bullet \bullet)$ \\
\hline Partial product bits & & $\bullet \bullet \bullet \bullet\left(\mathrm{B}_{1} \mathrm{~B}_{0}\right)_{2} \mathrm{~A} 4^{0}$ \\
& & $\bullet \bullet \bullet \bullet\left(\mathrm{B}_{3} \mathrm{~B}_{2}\right)_{2} \mathrm{~A} 4^{1}$ \\
Product & $\mathrm{P}=$ & $\bullet \bullet \bullet \bullet \bullet \bullet \bullet$
\end{tabular}

Radix-4 duplication in dab documentation.

It is showed up in the design above, growth is completed in radices 4 , in every movement, deficient thing stint 2 an ought to be designed, additional to aggregate fragmentary thing. While in radix-2 augmentation, each line of spots in the incomplete items network speaks to 0 or a moved variant of an absolute necessity be incorporated and included.

At initial, a " 0 " set as the benefit utmost bit of the multiplier. By before 3 bit of the multiplicand is recorded by table underneath and affording the going with condition:

$$
Z i=-2 x i+1+x i+x i-1
$$




\subsection{Correlation of Booth and Move and} Include Techniques

The Figure 7 shows that the full definition of multiplication methods.

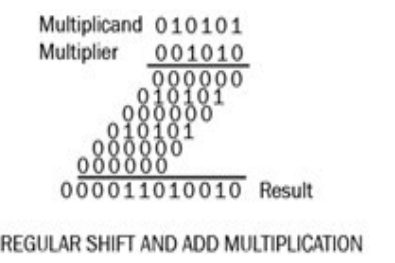

$$
\begin{aligned}
& \text { Multiplicand } 010101 \\
& \text { Multiplier } \quad 001010 \\
& \frac{\frac{01010}{111}}{\text { Intermediate }} \\
& 000001010 \\
& 00001010 \\
& \overline{000011010010} \text { Result } \\
& \text { BOOTH MULTIPLICATION }
\end{aligned}
$$

Figure 7. Multiplication method.

\subsection{Equipment usage of Booth}

Once the fragmented things are delivered then the development technique is on a very basic level the same as the display multiplier. Typically convey spare address are utilized with the last whole included utilizing a CRA.

Meanwhile the Booth Method put on to 2's supplement number juggling, mind is taken to ensure sign extensions is set up showed up touches in going with figure. For this situation, the multiplicand was balanced one piece to one side to go the viper while the low-arrange multiplicand position 0 is included. The Figure 8 defines about the Hardware Implimentation.

Every time the incomplete item will be moved no good position to one side and the sign reached one side.

Multiplicand moved left one piece spot which is tantamount on the way to copying by 2 .

A few procedures exist that decreases this undertaking with instant layouts. Once the table of the fractional items are drawn, every one of the columns of the halfway items must be mathematically reached out to $2^{\star} \mathrm{N}, \mathrm{N}$ - extent of
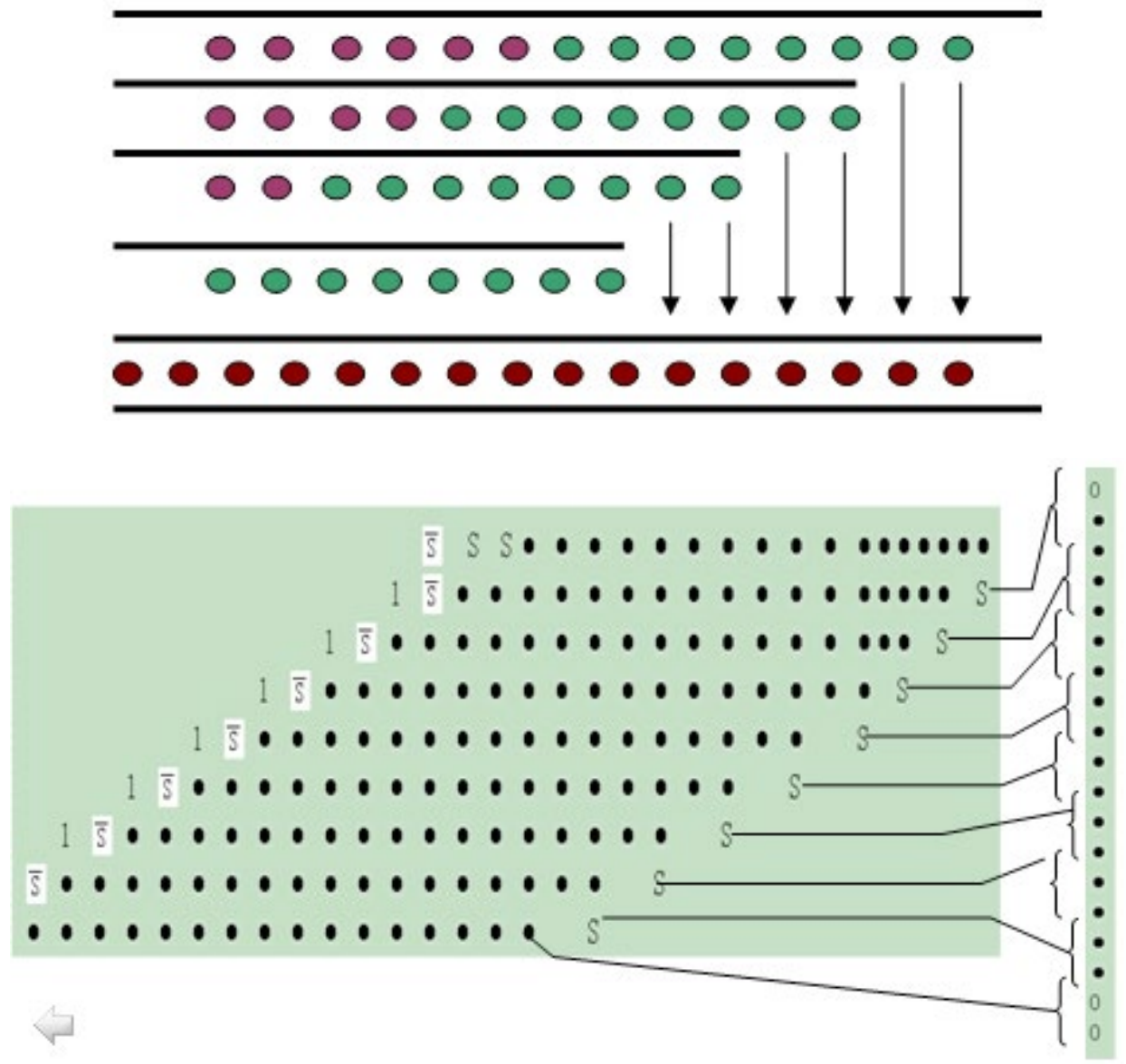

Figure 8. Hardware Implementation of Booth. 


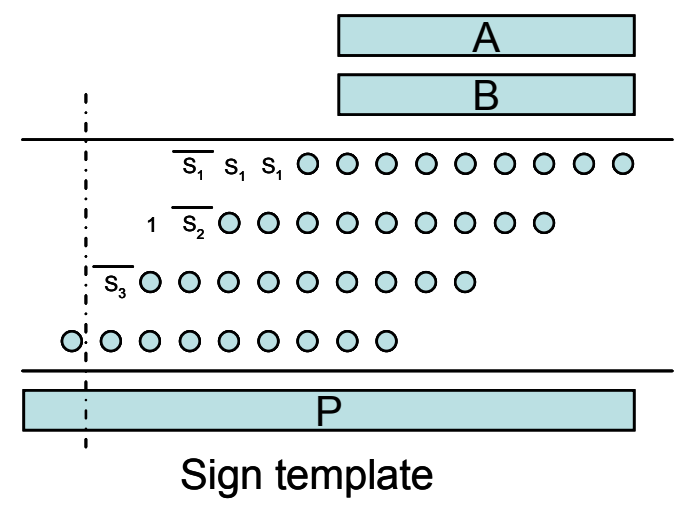

Figure 9. Sign template and sign extension.

the multiplicand. It is important for acquire amend comes about however it expands the capacitive load, the region and the computational time the layout, there are 16 bit numbers. What's more, seventeenth piece sign piece.

Likewise, fractional items on every column are entered l'complement numbers. The Figure 9 shows the Sign template and sign extension. On the off chance that 2'complement numbers are utilized then the $\mathrm{S}$ sections on the correct side can be expelled. If it's not too much trouble take note of that $\mathrm{S}$ digit is the sign piece of the stall encoding of that column)

\section{Conclusion}

In this paper, we proposed the fractional item aperture procedure for creating inexact equipment multipliers. The proposed strategy precludes various incomplete items empowering high zone and power funds while holding high exactness. Through a thorough mistake investigation, we scientifically described the actuated blunder measurements demonstrating that the mistake is limited and unsurprising and we proposed two blunder rectification techniques that exchange a little increment in control for high mistake training. We investigated item puncturing on an extensive arrangement of multiplier designs, assessing its effect on various models and mistake limits. In contrast with the best in class guess procedures, we

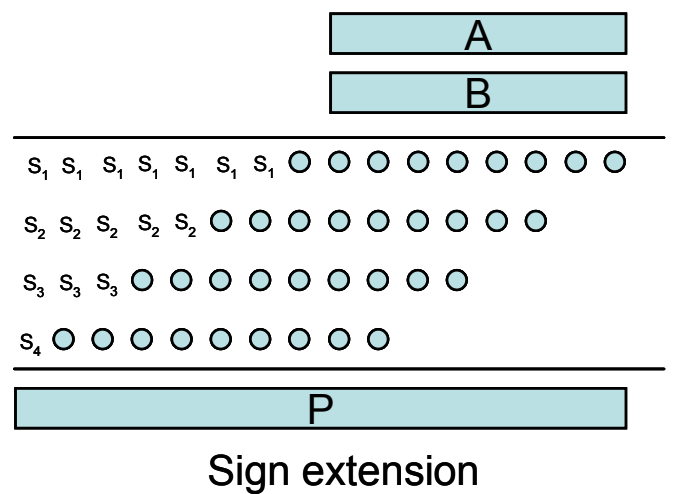

demonstrated that the proposed approach accomplishes critical picks up in power, region, and quality measurements of picture preparing and information investigation calculation.

\section{References}

1. Liu Y, Zhang T, Parhi KK. Computation error analysis in digital signal processing systems with over scaled supply voltage. IEEE Transactions on Very Large Scale Integration (VLSI) Systems. 2010; 18(94):517-26.

2. Lingamneni A, Enz C, Palem K, Piguet C. Synthesizing parsimonious inexact circuits through probabilistic design technique. ACM Transactions on Embedded Computing Systems. 2013; 12(2).

3. Momeni A, Han J, Montuschi P, Lombardi F. Design and analysis of approximate compressors for multiplication. IEEE Transactions on Computers. 2015; 64(4):984-94.

4. Zhu N, Goh WL, Zhang W, Yeo KS, Kong ZH. Design of low-power high-speed truncation-error-tolerant adder and its application in digital signal processing. IEEE Transactions on Very Large Scale Integration (VLSI) Systems. 2010; 18(8):1225-9.

5. Narayanamoorthy S, Moghaddam HA, Liu Z, Park T, Kim NS. Energy-efficient approximate multiplication for digital signal processing and classification applications. IEEE Transactions on Very Large Scale Integration (VLSI) System. 2015; 23(6):1180-4. 